\title{
Pediatric urological surgery readiness condition (PedsUROCON)
}

\author{
Roseanne Ferreira, $M D^{1}$; Daniel Keefe, $M D^{2}$; Yuding Wang, $M D^{3}$; Melissa McGrath, BASc ${ }^{1,3}$; \\ Martin A. Koyle, MD, MSc'; D. Robert Siemens, MD'; Luis H. Braga, MD, MSc, PhD ${ }^{1,3}$
}

'Clinical Urology Research Enterprise (CURE) Program, McMaster Children's Hospital, Hamilton, ON, Canada; ${ }^{2 T h e}$ Hospital for Sick Children, Department of Urology, Toronto, ON, Canada; ${ }^{3}$ Division of Urology, Department of Surgery, McMaster University, Hamilton, ON, Canada; 'Department of Urology, Queen's University, Kingston, ON, Canada

Cite as: Can Urol Assoc J 2020;14(7):E290-3. htrp://dx.doi.org/10.5489/cuai.6704

$\Lambda$ s of March 11, 2020, the World Health Organization declared coronavirus disease 2019 (COVID-19) a pandemic and urged countries to implement protocols to contain the virus spread. ${ }^{1}$ It was soon realized that the world was ill-prepared and too few lessons had been learned from previous infectious outbreaks in 2003 and 2009. ${ }^{2}$ As a result, preparation and planning were insufficient to deal with the impact of this strain on the healthcare system.

To deliver the optimal care despite this pervasive and fast-evolving event, healthcare managers must address and adjust four main domains in order to reach optimal surge capacity: maximize use of hospital structure, increase staff capacity, prioritize use of equipment and supplies, and develop an efficient communication system between hospitals. ${ }^{3,4}$ Another key factor in pandemic preparedness is the rapid acquisition of information on pathogen epidemiology. All of this knowledge is essential to make adjustments, which can increase the reliability of the current protocol, in addition to clarifying existing hospital vulnerabilities. More importantly, it can assist in predicting what further measures might be necessary to accommodate the influx of patients. ${ }^{3,4}$

It is important to recognize that patient flow plays a primary role in organization of hospital resources. ${ }^{4}$ Critically analyzing the needs and requirements of each healthcare component, by identifying a hierarchy of their services, can lead to hospital planning being more efficient, resulting in quicker establishment and mobilization of rapid-response groups. ${ }^{3,4}$ However, accuracy of communication is a major challenge identified when creating a mass protocol, as inconsistent terminology or triggers can generate confusion. ${ }^{4}$ For this reason, assuring optimal communication and networking between local and regional hospitals aids hospital management by allowing patient-tracking, information-sharing, and appropriate distribution of patients and supplies..$^{3-5}$

Defense readiness condition (DEFCON) is a system created by the U.S. military to standardize the level of reac- tion required for a threat level perceived by the military. ${ }^{6}$ DEFCON is graded from $1-5$, where 5 represents normal peacetime readiness and 1 represents maximum readiness - nuclear war is imminent. ${ }^{6}$ Each level is detailed with the expected actions and clearly states the trigger to activate the next level. This system establishes a clear and well-defined approach to assess readiness and could be used as a template in healthcare for crisis management. To achieve this, hospital departments should create protocols that prioritize procedures based on hierarchy and formally establish triggers that guide personnel when upgrading or downgrading readiness levels.

The concept of using the DEFCON model to create a crisis standard of care was previously suggested by Dr. Thomas Forbes from the Vascular Surgery Department at the University of Toronto when designing the VASCCON. ${ }^{7}$ Based on this experience, we propose the pediatric urological surgery readiness condition (PedsUROCON). PedsUROCON includes guidance for different alertness levels ranging from a fully functional hospital capacity (level 5) to when maximal hospital capacity has been reached, and even life/ limb-threatening surgeries cannot be performed (level 1). The purpose of this decision-making algorithm is to serve as a template for long-term pediatric urology pandemic/ emergency preparedness. Fig. 1 describes which procedures should be restricted once each level of alertness is reached.

PedsUROCON alertness levels should be transitioned (i.e., upgraded or downgraded) once hospital capacity reaches predetermined thresholds. We suggest $40 \%, 60 \%$, $80 \%$, and $100 \%$ of hospital capacity (Table 1 ). Hospital capacity should be determined by hospital administrators when factoring physical space, personnel availability, resources, and supplies. Regional communication and networking are paramount for this system to be effective. Constant communication must be maintained throughout the crisis to coordinate the efficient flow of patients, resources, and information, with the goal of reducing the need to escalate any healthcare institution to the next PedsUROCON level. 


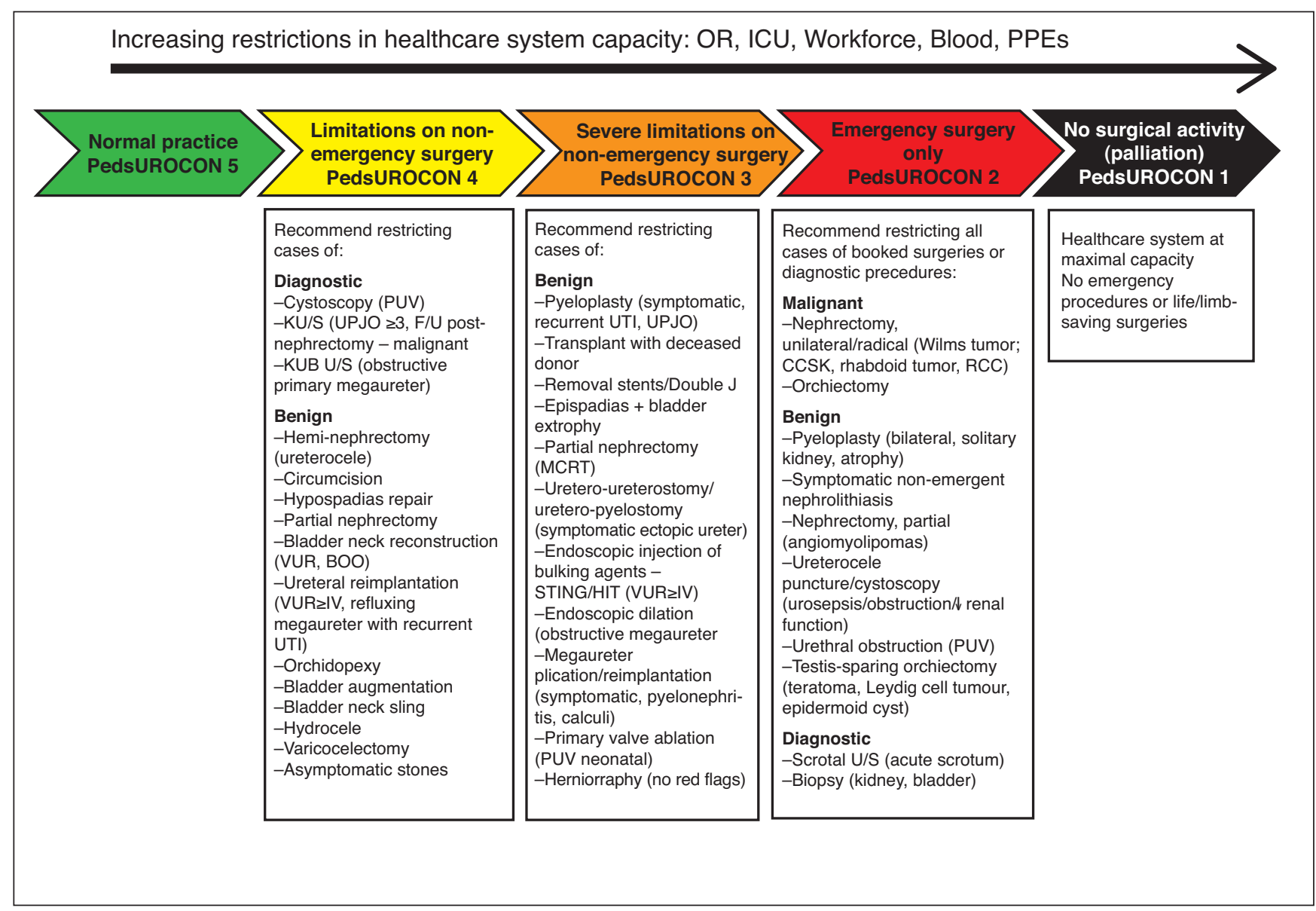

Fig. 1. Pediatric urological surgery readiness condition (PedsUROCON). BOO: bladder outlet obstruction; CCSK: clear-cell sarcoma of the kidney; HIT: hydrodistention implantation technique; ICU: intensive case unit; KU/S: kidney ultrasound; KUBU/S: kidney ureter bladder ultrasound; MCRT: mutliocular cystic real tumor; OR: operating room; PPE: personal protective equipment; PUV: posterior urethral valves; RCC: renal cell carcinoma; RLND: retroperitoneal lymph node dissection; STING: subureteral teflon injection; UTI: urinary tract inflammation; VUR: vesicoureteral reflux.

Each level of PedsUROCON can be implemented on the fast-developing COVID-19 outbreak (Fig. 2). The protocol is based on resources and procedures offered at our institution. We recommend adjusting thresholds for triggers based on regional epidemiological forecasting, hospital programs and specialized care areas, physical capacity, and services offered by the local healthcare system. Lastly, for optimizing functionality and readiness, this system should be updated and tested annually, after a major change in clinical care protocols or major renovations at a hospital - whichever comes first.

In order to achieve maximum preparedness, we advise each hospital to adopt the proposed algorithm on a departmental level (i.e., Urology, Medicine, Emergency, etc.); when integrated, the PedsUROCON decision matrix will clearly outline prioritization requirements for hospital services and procedures. The adoption of this algorithm will further aid in establishing intra-hospital flow by equalizing triggers and aid in anticipated economical challenges by quantifying demand based on the appropriate PedsUROCON level (Table 2). Furthermore, it will assist departments to systematically triage and, as the pandemic stabilizes, advocate for priority of cases, as well as equitable resource distribution between departments.

\section{Conclusions}

Creating a surge capacity mitigation protocol involves a multilayered level of interconnectivity between local hospitals, regional healthcare services, hospital supplies management, and emergency services infrastructure. Current protocols are lacking and inefficient in being able to deal with events such as the COVID-19 outbreak. Establishing the PedsUROCON protocol will allow hospitals and healthcare infrastructures to be forewarned, thus allowing them to be forearmed.

Competing interests: The authors report no competing personal or financial interests related to this work.

This paper has been peer-reviewed 


\begin{tabular}{|c|c|c|}
\hline Alertness level & $\begin{array}{l}\text { Thresholds for } \\
\text { action } \\
\text { (hospital maximal } \\
\text { capacity, } \\
\% \text { occupancy) }\end{array}$ & Clinical significance \\
\hline PedsUROCON 5 & $<40 \%$ & Normal practice \\
\hline PedsUROCON 4 & $40-59 \%$ & $\begin{array}{l}\text { Limitations on non- } \\
\text { emergency surgery } \\
\text { Booked cases limited to } \\
\text { symptomatic stones (pain } \\
\text { from renal colic, non- } \\
\text { obstructing) }\end{array}$ \\
\hline PedsUROCON 3 & $60-79 \%$ & $\begin{array}{l}\text { Severe limitations on } \\
\text { non-emergency surgery } \\
\text { Booked cases limited } \\
\text { to obstructing stones } \\
\text { (severe hydronephrosis) }\end{array}$ \\
\hline PedsUROCON 2 & $80-99 \%$ & $\begin{array}{c}\text { Emergency surgeries } \\
\text { only } \\
\text { No booked cases, } \\
\text { emergency cases, } \\
\text { including septic stones } \\
\text { or increased creatinine } \\
\text { (acute kidney injury), } \\
\text { consideration for } \\
\text { nephrostomy tube } \\
\text { insertion }\end{array}$ \\
\hline PedsUROCON 1 & $100 \%$ & $\begin{array}{c}\text { No surgical activity } \\
\text { (palliation) } \\
\text { No emergent cases } \\
\text { (patients with septic } \\
\text { stones are offered } \\
\text { palliative care - IV } \\
\text { antibiotics and pain } \\
\text { medications) }\end{array}$ \\
\hline
\end{tabular}

\section{Summary}

Purpose: Long-term pediatric urology pandemic plan for patient flow management. PedsUROCON alertness levels should be transitioned once hospital capacity reaches specific thresholds. Hospital should communicate with the network of regional hospitals to organize patient flow. Alertness on patient flow facilitates prioritization of equipment, structure, and services.

Thresholds for action: PedsUROCON 5: $<40 \%$ occupancy of hospital maximal capacity; PedsUROCON 4: 40-59\%; PedsUROCON 3: 60-79\%; PedsUROCON 2: 80-99\%; and PedsUROCON 1: $\geq 100 \%$ of occupancy.

Maintenance: Based on the current structure of the hospitals and demands, their PedsUROCON should be updated yearly, following a major protocol change or after a major hospital renovation - whichever comes first.

Goals: 1) Organize patient flow in pediatric urology; 2) standardize communication between hospital departments to optimize resource use; 3) promote communication between hospitals to improve pandemic/emergency planning and reduce stress points in regional healthcare services.

\section{Being forewarned is being forearmed.}
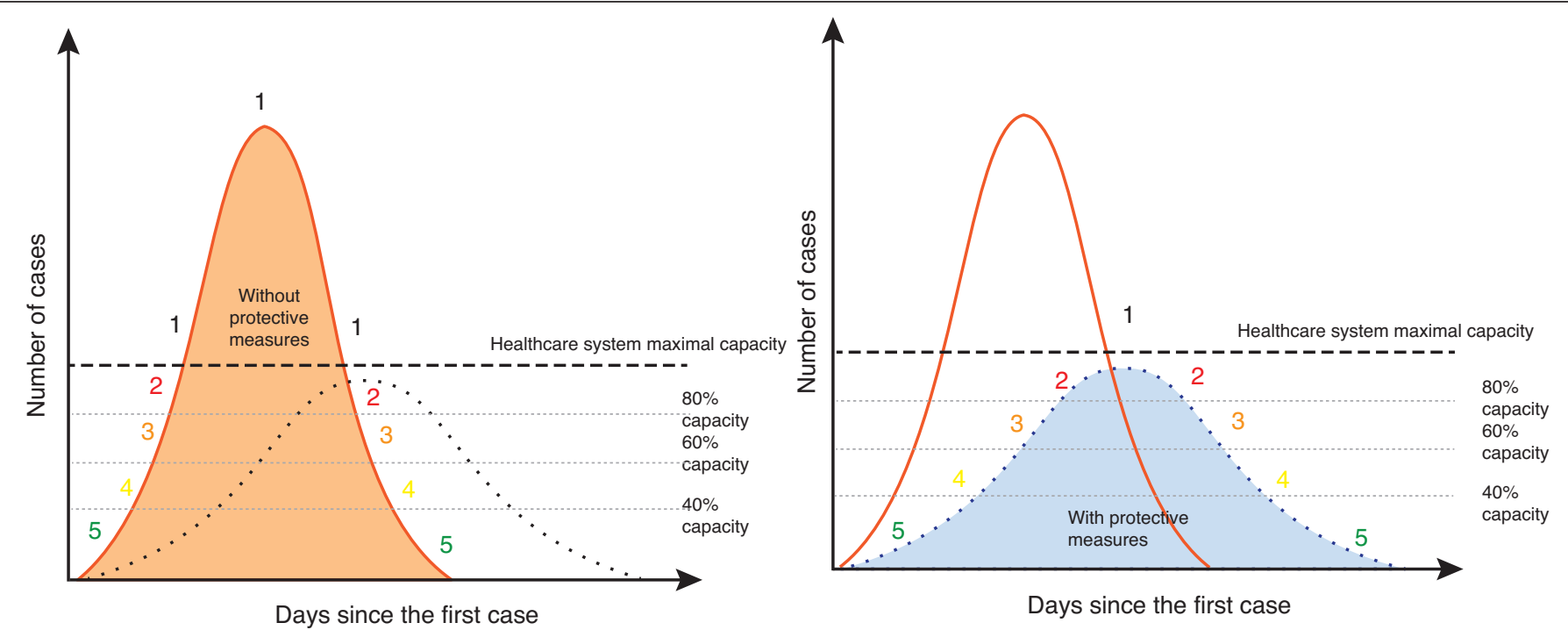

Fig. 2. PedsUROCON application in COVID-19 pandemic situation. 


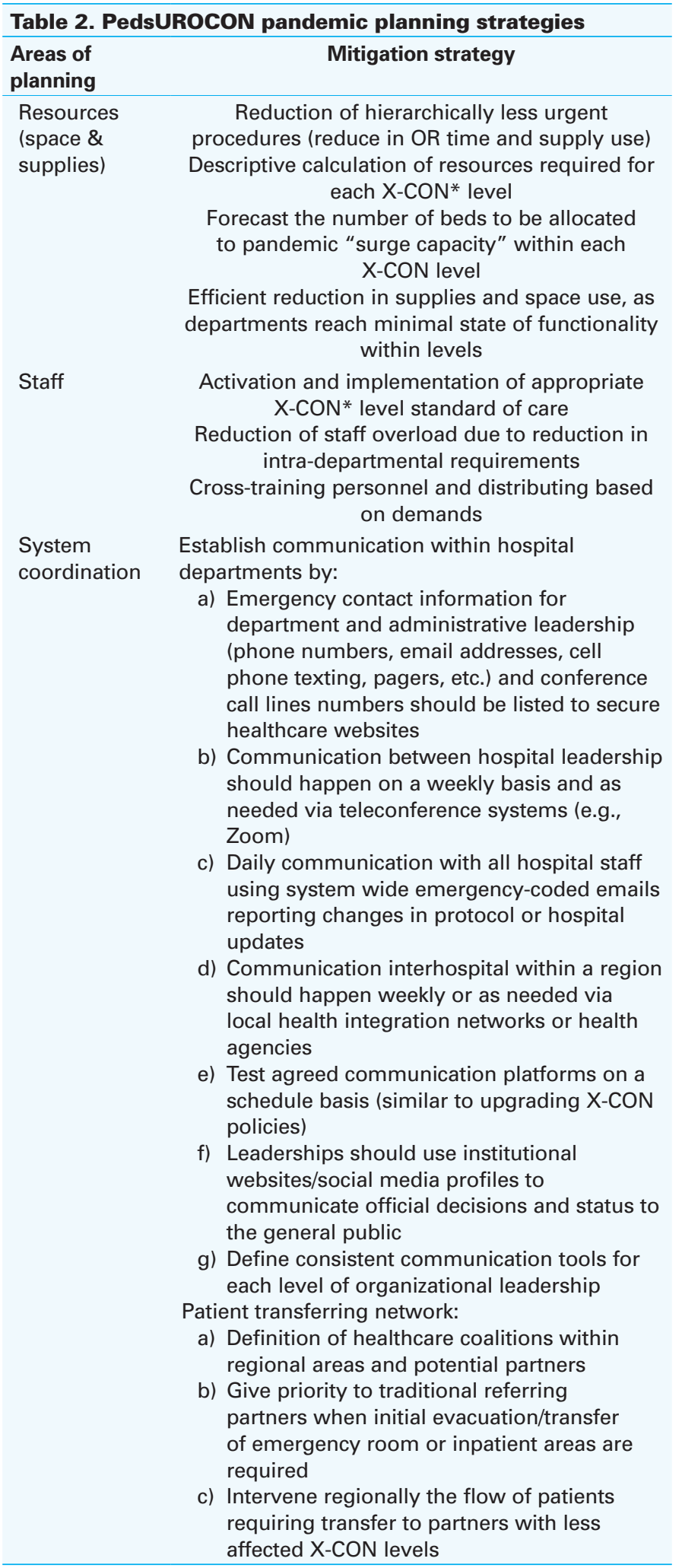

*X-CON refers to the readiness condition of a given department/specialty. Adapted from Dichter et al. ${ }^{5}$

\section{References}

1. World Health Organization. Coronavirus disease (COVID-2019) situation reports. Available at: https:// www.who.int/dg/speeches/detail/who-director-general-s-opening-remarks-at-he-media-briefing-on-covid19-11-march-2020. Accessed March 2020.

2. Peeri NC, Shrestha N, Rahman MS, et al. The SARS, MERS, and novel coronavirus (COVID-19) epidemics, the newest and biggest global health threats: What lessons have we learned? Int I Epidemiol 2020 Feb 22. https://doi.org/10.1093/iie/dyau033

3. Sheikhbardsiri H, Raeisi AR, Nekoei-moghadam M, et al. Surge capacity of hospitals in emergencies and disasters with a preparedness approach: A systematic review. Disaster Med Public Health Prep 2017;11:612-20. https://doi.org/10.1017/dmp.2016.178

4. Hamele M, Neumayer K, Sweney J, et al. Always ready, always prepared-preparing for the next pandemic. Transl Pediatr 2018;7:344-55. hitps://doi.org/10.21037/tp.2018.09.06

5. Dichter JR, Kanter RK, Dries D et al; Task Force for Mass Critical Care. System-level planning, coordination, and communication: Care of the critically ill and injured during pandemics and disasters: CHEST consensus statement. Chest 2014;146:e87S-e102S. https://doi.org/10.1378/chest.14-0738

6. Cold War Gallery Overview. National Museum of The United States Air Force. Available at: https://www. nationalmuseum.af.mil/Visit/Museum-Exhibits/Fact-Sheets/Display/Article/197649/cold-war-galleryoverview/ June 01, 2015. Accessed April 3, 2020.

7. @IL_Forbes (Thomas Forbes). I hope everyone is trying to stay safe (and sane) during these crazy times, no matter where you are on the COVID19 curve or journey. Some have asked me for more info re VASCCON. Feel free to use the attached slides if you find them helpful. 1/2. Twitter, 28 Mar 2020, https://twitter.com/TL_Forbes/status/1243965386250489856?s=20

Correspondence: Dr. Luis H. Braga, Division of Urology, Department of Surgery, McMaster University, Hamilton, 0N, Canada; braga@memaster.ca 